\title{
Qatar Biobank: A Paradigm of Translating Biobank Science into Evidence-Based Health Care Interventions
}

\author{
Eleni Fthenou, Asma Al Thani, ${ }^{1-4}$ Ajaeb Al Marri, ${ }^{5}$ and Nahla Afifi ${ }^{1}$
}

\begin{abstract}
Biobank science is progressively becoming indispensable for the development of novel diagnostic tools in health care, by pairing standardized high-quality biological samples, phenotypic, and omics data required to best characterize the underlying biological mechanisms of response to therapy and survival. Qatar Biobank (QBB), Qatar's National Repository Centre for biological samples and health information, aligning with these endeavors, has developed a strategic framework to enable biobanking science that can be transformed into tangible health care diagnostic tools. In this concept, QBB works closely with multidisciplinary stakeholders: (1) governmental authorities $(n=2),(2)$ health providers $(n=3),(3)$ academic institutions $(n=28)$, (4) other research institutions $(n=6)$, and (5) the Qatar National Research Fund, by providing data and biospecimens to research projects. The local community organizes campaigns through social media and interactive events, spreading the concept of biobanking to inform and encourage people to participate. Up to now, QBB has recruited up to $30 \%$ of the targeted population and collaborated with 35 national and international research entities contributing to more than 170 research projects. QBB is referring about 53\% of its participants to the Hamad general hospital through the integrated information system, revealing the need of new health screening approaches for public health policy makers. QBB contributed to the development of a customized genetic screening microarray tailored to the Qatari population that will be used in research as well as for the screening of variants of medical relevance in Qatar, promoting precision medicine in Qatar's health care system. The QBB strategic plan is a paradigm for the optimal utilization of resources, infrastructure, and investments engaging the local authorities and the research entities, and committing them to data sharing and working together toward the discovery of evidence-based health care interventions.
\end{abstract}

Keywords: Qatar Biobank, biobanking, health care, precision medicine

\section{Introduction}

I

$\mathrm{N}$ RECENT YEARS, advances in genomics coinciding with the emergence of other large-scale high-throughput "omics" technologies and the development of bioinformatics capacities and tools for handling large data sets have given new insights into biomarker research endeavors and the understanding of the underlying molecular mechanisms of the origin of health and diseases. ${ }^{1}$ One of the major issues in biomarker discovery remains the heterogeneity inherited due to the disease itself as the disease evolves over time. Consequently, clinically collected specimens carry disease-inherited changes in the molecular profiles of patients, modifying the effect and thus the outcome of research.

Longitudinal prospective cohort studies are conceptually suitable for the discovery of novel biomarkers through collec- tion of adequate data and biological samples before (preclinical), during (clinical), and after disease development., ${ }^{2,3}$ The identification of novel biomarkers that can predict treatment response at the patient individual level requires the standardized collection, processing, and storage of biological samples. Biobanking and the new field of biospecimen science have significantly contributed to generating reproducible results in biomedical research. ${ }^{4}$ Biobank science and the unbiased largescale approaches such as omics can leverage the discovery of novel predictive biomarkers in the field of precision medicine.

The Qatar Biobank for Medical Research (QBB) was established by Qatar Foundation for Education, Science and Community Development in collaboration with the Ministry of Public Health and Hamad Medical Corporation in 2012. It was an effort of the Qatar government to empower research

\footnotetext{
${ }^{1}$ Qatar Biobank, Qatar Foundation for Education, Science, and Community, Doha, Qatar.

${ }^{2}$ College of Health Sciences and ${ }^{3}$ Biomedical Research Center, Qatar University, Doha, Qatar.

${ }^{4}$ Qatar Genome Program Committee, Doha, Qatar.

${ }^{5}$ Hamad Medical Corporation, Doha, Qatar.
} 
toward the discovery and development of novel health care interventions, specifically in the field of precision medicine. $^{5,6}$ QBB is a research enterprise platform across Qatar, acting as the National Repository Centre for biological samples and health information records and making them available to the research community in Qatar and worldwide.

QBB has a unique structure compared with other biobanks worldwide, since it is a center by itself and not integrated within any academic institution or hospital. QBB as a national biorepository depends mainly on governmental authority support and their understanding of the biobank's contribution to the local society.

QBB is working closely with stakeholders to achieve its vision of delivering the resources to the research community to achieve outstanding improvement in diagnostic and prognostic intelligence required to deliver personalized health care for the benefits of people in Qatar, the region and worldwide. In this article, we present QBB's framework, aiming to set up a multicenter/multidisciplinary initiative translating biobank science into evidence-based health care interventions.

\section{QBB Operational Plan for Biobank-Dependent Evidence-Based Health Care Interventions}

Currently, QBB has recruited more than 20,000 participants aiming to reach the target of 60,000 Qataris and longterm residents (have resided in Qatar $>18$ years). The QBB biorepository holds more than two million biological samples (blood, saliva, urine, and derives) and the data repository covers most of the molecular profiles of the participants providing data on genomics, transcriptomics, proteomics, metabolomics, and epigenomics with deep phenotypic profile and imaging data (MRI, carotid ultrasound, bone density through iDexa, and retina scan). ${ }^{5,6}$ QBB achieved the main goal to enroll a sufficient number of participants $(>30 \%$ of the targeted population) and implemented a coordinated operational plan dedicated to bring together multidisciplinary stakeholders promoting biobank-dependent research to cultivate evidence-based health care interventions. The QBB operational plan (Fig. 1) covers a concrete network bringing together stakeholders across the country and international experts to enhance our effort to translate QBB data into tangible health care interventions. QBB collaborates with research and research-related stakeholders including governmental authorities, health care providers, academic entities, and other research institutions.

\section{QBB impact on governmental level: policies, regulations, and guidelines}

QBB works together with the Qatar Ministry of Public Health (MoPH) and the Ministry of Municipality and Environment, providing data to be utilized by policy makers to develop evidence-based policies, regulations, and guidelines for the protection of public health and the environment in Qatar. QBB has actively participated in structured discussions with the MoPH related to the Islamic ethical principles of biomedical research as well as the common ethical challenges on participants' autonomy, protection, and data confidentiality in biobanks. The Islamic aspects of related ethical challenges in biobank science are poorly studied. ${ }^{7}$ QBB supported the development of national documents such as the Guidance for the Use of Stored Data and Biological Specimens in Human Research and Guidance for the Design, Ethical Review, and Conduct of Genomic Research in Qatar, published by the MoPH, ${ }^{8}$ by providing data reflecting the Qatar's society ethos and morals related to medical research and biobanking. In addition QBB is part of the MoPH committees in Mental Health Strategy, National Cancer Strategy, Qatar National Diabetes Strategy and the Qatar National Dementia Plan. ${ }^{9}$ Moreover, QBB is linked with Qatar's National Medical Information System (Cerner), referring $\sim 53 \%$ of its participants to the Hamad General Hospital, the main public hospital in Qatar. This has placed QBB, not intentionally, as an additional screening center for the Qatari population, adding to the disease prevention programs of the MoPH and Primary Health Centers Corporation. QBB has also participated in the development of the Qatar National Air Quality standards in a call from the Qatar Ministry of Municipality and Environment, providing both expertise and data.

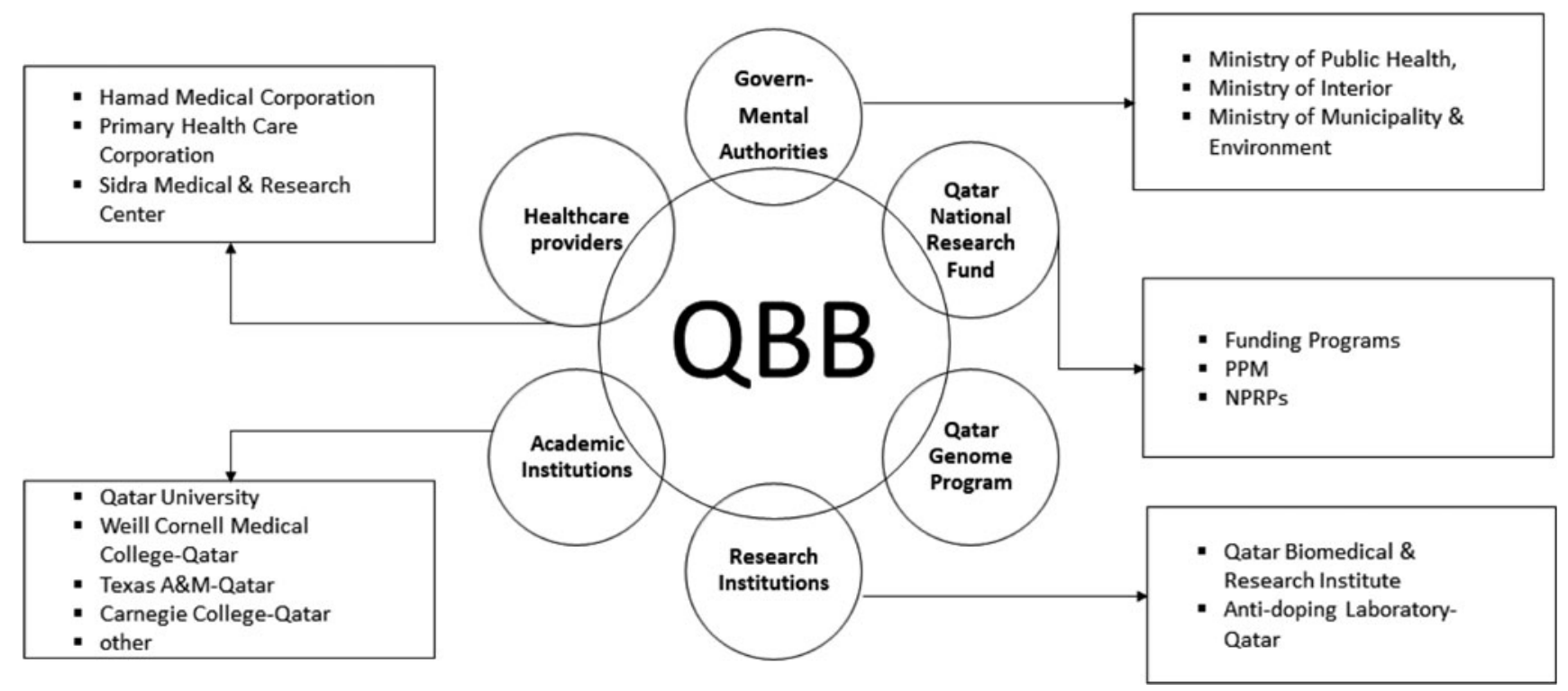

FIG. 1. Qatar Biobank operational plan—collaboration with multidisciplinary stakeholders. 


\section{QBB impact on Qatar's capacity building}

QBB, recognizing the importance of cultivate health care research for the next generation, is promoting Qatar's capacity building by supporting academic students providing data (free of charge) to be utilized for educational projects for both undergraduate $(n=26)$ and postgraduate theses $(\mathrm{PhDs}, n=3$; MScs, $n=5$ ). Moreover, QBB collaborates with the College of Health Sciences at Qatar University under the Sciences Education and the Human Health Activity program with the Ministry of Education and Higher Education, by introducing biobank science and health research to high school students.

\section{QBB impact on personalized medicine}

Precision medicine is progressively becoming a reality. QBB works toward the quality and standardization of biological samples acquisition required to best characterize and track disease onset and evolution as well as to understand the underlying biological mechanisms of response to therapy and survival. QBB follows national and international best practices such as the International Society for Biological and Environmental Repositories (ISBER's) best practices along with the Quality Management ISO 9001 standard and Information Security Management System ISO 27001 accreditation and is engaged in advancing research and personalized medicine in Qatar and worldwide.

More specifically, QBB is translating biobank science into health care interventions by working closely with other stakeholders such as the Qatar Genome Program, Weil Cornel Medicine-Qatar (WCM-Q), and Hamad Medical Corporation (HMC), to develop a genetic screening microarray tailored to the Qatari population (Qatari gene chip or Q-chip). The Q-chip was customized for research as well as for the screening of variants of medical relevance in Qatar compared with the existing practice of low-throughput screening of a few variants or high-throughput screening using a commercial array. Currently, QBB laboratories are engaged in the process of the Q-chip content research and Q-chip content clinical validation and collecting College of American Pathologists (CAP) requirements to apply for accreditation. The Q-chip is in the stage of validation as a screening tool for a number of diseases. QBB is also aiming to provide personal genomic consultation based on whole genome sequence data and the Q-chip data. As a first step, the genomic consultation will provide participants with genomic information and recommendations on general health and wellness, diet, exercise, and some metabolic conditions such as diabetes. In its later stages, QBB will be validating the response and acceptance of the participants related to the genomic consultation, and will move forward to provide genomic consultation on pharmacogenomics of specific drugs and possibly polygenic risk scores becoming part of precision medicine practice in Qatar. Precision medicine services delivered by $\mathrm{QBB}$ have been designed to be a part of a more comprehensive strategy in close collaboration with health care providers such HMC. This is one of the QBB's ambitious endeavors toward the future of medicine, wherein precision medicine flips the script of conventional medicine.

\section{Conclusion}

The potential for new discoveries in the field of precision medicine is growing exponentially. Researchers, around the world, have developed diagnostic tools using new emerging technologies by pairing biological and genomic data. QBB, aligning with these endeavors, has developed a strategic framework to enable biobanking science transformed into tangible health care diagnostic tools by the generation of comprehensive biological and molecular research data collected longitudinally. QBB is suggesting a paradigm on how to bring together multidisciplinary stakeholders and commit them to data sharing to move forward with the discovery of novel health care interventions.

QBB shares data along with biological material and imaging data for medical research and development projects. The data sharing and biological samples process is conducted in line with QBB policies and regulations. Researchers interested in accessing QBB data and/or biological samples should submit a research project application form to QBB Research Access Office using the link (www.qatarbiobank.org.qa).

\section{Author Disclosure Statement}

No conflicting financial interests exist.

\section{Funding Information}

There was no funding.

\section{References}

1. Chen R, Snyder M. Promise of personalized omics to precision medicine. Wiley Interdiscip Rev Syst Biol Med 2013;5:73-82.

2. Vineis $P$, Chadeau-Hyam M. Integrating biomarkers into molecular epidemiological studies. Curr Opin Oncol 2011; 23:100-105.

3. Yang L, Chen Y, Yu C, Shen B. Biobanks and their clinical application and informatics challenges. Adv Exp Med Biol 2016;939:241-257.

4. Vaught J. Biobanking comes of age: The transition to biospecimen science. Annu Rev Pharmacol Toxicol 2016;56:211-228.

5. Thani AA, Fthenou E, Paparrodopoulos S, et al. Qatar Biobank cohort study: Study design and first results. Am J Epidemiol 2019 [Epub ahead of print]; DOI: 10.1093/ aje/kwz084.

6. Al Kuwari H, Al Thani A, Al Marri A, et al. The Qatar Biobank: Background and methods. BMC Public Health 2015;15:1208.

7. Alahmad G, Dierickx K. Ethics of research biobanks: Islamic perspectives. Biopreserv Biobank 2018 [Epub ahead of print]; DOI: 10.1089/bio.2017.0067.

8. Health MoP. Research Doha, Qatar2019. https://www.moph .gov.qa/about-us/Pages/research.aspx (accessed May 20, 2019).

9. Health QMoP. National health strategy 2018-2022, Qatar Ministry of Public Health, 2018. https://www.moph.gov.qa/ HSF/Documents/short\%20report\%20eng\%2020.03.2018.pdf (accessed May 20, 2019).

Address correspondence to: Eleni Fthenou, PhD Qatar Biobank Qatar Foundation for Education, Science, and Community

Building 317, Hamad Medical City Doha 9744

Qatar

E-mail: efthenou@qf.org.qa 